\title{
Evaluation of reference gene suitability for quantitative expression analysis by quantitative polymerase chain reaction in the mandibular condyle of sheep
}

\author{
XIN JIANG ${ }^{1,2^{*}}$, YANG XUE $^{3 *}$, HONGZHI ZHOU $^{1}$, SHOUHONG LI $^{2}$, ZONGMIN ZHANG $^{1}$, \\ RUI HOU ${ }^{1}$, YUXIANG DING ${ }^{1}$ and KAIJIN HU ${ }^{1}$
}

\begin{abstract}
${ }^{1}$ State Key Laboratory of Military Stomatology, Department of Oral and Maxillofacial Surgery, School of Stomatology,
The Fourth Military Medical University, Xi'an, Shaanxi 710032; ${ }^{2}$ Department of Oral and Maxillofacial Surgery, Dongfeng Hospital, Hubei University of Medicine, Shiyan, Hubei 442008; ${ }^{3}$ State Key Laboratory of Military Stomatology, Department of Oral Biology, School of Stomatology, The Fourth Military Medical University, Xi'an, Shaanxi 710032, P.R. China
\end{abstract}

Received July 13, 2014; Accepted May 8, 2015

DOI: $10.3892 / \mathrm{mmr} .2015 .4128$

\begin{abstract}
Reference genes are commonly used as a reliable approach to normalize the results of quantitative polymerase chain reaction (qPCR), and to reduce errors in the relative quantification of gene expression. Suitable reference genes belonging to numerous functional classes have been identified for various types of species and tissue. However, little is currently known regarding the most suitable reference genes for bone, specifically for the sheep mandibular condyle. Sheep are important for the study of human bone diseases, particularly for temporomandibular diseases. The present study aimed to identify a set of reference genes suitable for the normalization of qPCR data from the mandibular condyle of sheep. A total of 12 reference genes belonging to various functional classes were selected, and the expression stability of the reference genes was determined in both the normal and fractured area of the sheep mandibular condyle. RefFinder, which integrates the following currently available computational algorithms: geNorm, NormFinder, BestKeeper, and the comparative $\Delta \mathrm{Ct}$ method, was used to compare and rank the candidate reference genes. The results obtained from the four methods demonstrated a similar trend: RPL19, ACTB, and PGK1 were the most stably expressed reference genes in the sheep mandibular condyle. As determined by RefFinder comprehensive analysis,
\end{abstract}

Correspondence to: Professor Kaijin Hu, State Key Laboratory of Military Stomatology, Department of Oral and Maxillofacial Surgery, School of Stomatology, The Fourth Military Medical University, 145 West Changle Road, Xi'an, Shaanxi 710032, P.R. China

E-mail: hukaijin@fmmu.edu.cn

*Contributed equally

Key words: reference gene, sheep, geNorm, NormFinder, BestKeeper, comparative $\Delta \mathrm{Ct}$ method the results of the present study suggested that RPL19 is the most suitable reference gene for studies associated with the sheep mandibular condyle. In addition, ACTB and PGK1 may be considered suitable alternatives.

\section{Introduction}

Reverse transcription-quantitative polymerase chain reaction (RT-qPCR) is commonly used to measure the expression levels of a selected gene of interest by quantifying the mRNA transcripts. RT-qPCR technology provides highly sensitive and accurate expression profiles $(1,2)$. However, numerous parameters need to be controlled in order to obtain reliable quantitative expression measurements. These parameters include variations in the initial sample amount, RNA recovery, RNA integrity, efficiency of cDNA synthesis, and differences in the overall transcriptional activity of the tissue or cells analyzed (3). Therefore, reference genes are commonly used as internal controls. The reference gene is submitted to the same experimental protocol as the target gene in order to normalize qPCR data, and in order to reduce possible errors generated during the quantification of gene expression. The expression levels of the target gene are then normalized according to the values of the internal controls. Therefore, selecting a suitable reference gene is important for the correct interpretation of RT-qPCR analyses. Typically, reference genes satisfy the following criteria: They are highly expressed in the cells of interest, variability in the expression levels of the reference gene between samples is minimal, and the reference gene expression is not influenced by experimental conditions $(2,4)$. However, it has recently become apparent that no single gene is constitutively expressed in all cell types and under all experimental conditions, implying that the expression stability of the intended control gene must be verified prior to each experiment. Therefore, the selection of a suitable reference gene is largely dependent on the experimental tissue samples.

At present, GAPDH, ACTB, and $18 S$ rRNA are the most commonly used reference genes. However, previous studies 
have demonstrated that these reference genes are not always suitable, as they exhibit different behaviors in various types of cells and tissue $(5,6)$, particularly in sheep. Due to their unique advantages (such as their similarity to humans in anatomy, physiology and wound repair mechanisms), sheep are widely used in medical experimental studies. Previous studies have successfully identified the most suitable reference genes for ovine nervous tissue, spleen, ileum, lung, mesenteric lymph node, pulmonary tissue, and blood (7-10). However, little is currently known regarding the most suitable reference genes for bone, specifically for the sheep mandibular condyle.

The present study aimed to identify a set of reference genes suitable for the normalization of sheep mandibular condyle RT-qPCR data. A total of 12 commonly-used reference genes belonging to various functional classes were selected, and their expression stability was determined in both normal and fractured sheep mandibular condyles. RefFinder was used to validate the reference genes.

\section{Materials and methods}

Animals. Nine healthy sheep (six male and three female; age, 1 year; weight, 30-40 kg) were included in the present study. The sheep were obtained from the Animal Experimental Center and housed at the School of Stomatology, both at The Fourth Military Medical University (Xi'an, China). Condylar fractures on the right side of the sheep mandibular were created as previously reported (11). Briefly, the zygomatic arch and the panniculus carnosus muscles at the surface of the capsule of the temporomandibular joint were exposed via a curved pre-auricular skin incision. A horizontal incision was subsequently made through the capsule at the condylar neck to open the inferior joint space. An oblique vertical osteotomy was made from the lateral pole of the condyle to the medial side of the condylar neck, using an ultrasound osteotome (Guilin Woodpecker Medical Instrument Co., Ltd. Guilin, China). The left side of the sheep mandibular served as a control. Three sheep were postoperatively sacrificed at the end of the second, fourth and twelfth week by dissecting the carotid artery (under a general anaesthetic) resulting in acute hemorrhagic death. The bones surrounding the fracture line on the right side, and the bones from the corresponding area on the left side were subsequently collected for further experimentation.

The present study was approved by the Ethics Committee of the School of Stomatology, The Fourth Military Medical University. All surgical procedures were conducted under satisfactory anesthesia $(0.1 \mathrm{ml} / \mathrm{kg}$ xylazine hydrochloride; Military Veterinary Research Institute of Military Medical Science, Changchun, China). The sheep were cared for according to the guidelines set by the Laboratory Animal Research Centre of the Fourth Military Medical University.

Total RNA extraction and cDNA synthesis. Total RNA was extracted using the Total RNA kit I (Omega Bio-Tek, Inc., Norcross, GA, USA), according to the manufacturer's instructions. Contaminating genomic DNA was removed by on-column treatment of each sample with DNase I (Omega Bio-Tek, Inc., Norcross, GA, USA). The purity and quality of the extracted RNA was evaluated using a Nanodrop 2000 spectrophotometer
Table I. Primer sequences of the 12 candidate reference genes.

\begin{tabular}{|c|c|}
\hline Gene name & Primer sequences \\
\hline \multirow[t]{2}{*}{ RPL19 } & F: 5' AGCCTGTGACTGTCCATTCC 3' \\
\hline & R: 5' ACGTTACCTTCTCGGGCATT 3' \\
\hline \multirow[t]{2}{*}{ YWHAZ } & F: 5' AGACGGAAGGTGCTGAGAAA 3' \\
\hline & R: 5' CGTTGGGGATCAAGAACTTT 3' \\
\hline \multirow[t]{2}{*}{$P G K 1$} & F: 5' ACTCCTTGCAGCCAGTTGCT 3' \\
\hline & R: 5' AGCACAAGCCTTCTCCACTTCT 3' \\
\hline \multirow[t]{2}{*}{$H P R T$} & F: 5' TTTATTCCTCATGGACTAATTATGGA 3 \\
\hline & R: 5' CCACCCATCTCCTTCATCAC 3' \\
\hline \multirow[t]{2}{*}{ TFRC } & F: 5' TTCTGGGCAGACCTCAAATC 3' \\
\hline & R: 5' CAGCTTCACGTGGGACATAA 3' \\
\hline \multirow[t]{2}{*}{$G 6 P D$} & F: 5' TGACCTATGGCAACCGATACAA 3' \\
\hline & R: 5' CCGCAAAAGACATCCAGGAT 3' \\
\hline \multirow[t]{2}{*}{$B 2 M$} & F: 5' CTGTCGCTGTCTGGACTGG 3' \\
\hline & R: 5' TTTGGCTTTCCATCTTCTGG 3' \\
\hline \multirow[t]{2}{*}{$A C T B$} & F: 5' CCAACCGTGAGAAGATGACC 3' \\
\hline & R: 5' CCAGAGGCGTACAGGGACAG 3' \\
\hline \multirow[t]{2}{*}{ SDHA } & F: 5' CATCCACTACATGACGGAGCA 3' \\
\hline & R: 5' ATCTTGCCATCTTCAGTTCTGCTA 3' \\
\hline \multirow[t]{2}{*}{ RPS 24} & F: 5' TTTGCCAGCACCAACGTTG 3' \\
\hline & R: 5' AAGGAACGCAAGAACAGAATGAA 3' \\
\hline \multirow[t]{2}{*}{$18 S$} & F: 5' TGGTCGCTCGCTCCTCTCC 3' \\
\hline & R: 5' CGCCTGCTGCCTTCCTTGG 3' \\
\hline \multirow[t]{2}{*}{ GAPDH } & F: 5' CCAGAGGCGGACTACTACG 3' \\
\hline & R: 5' CCG AGA GAGCAACACAGG 3' \\
\hline
\end{tabular}

F, forward; R, reverse.

(Thermo Fisher Scientific, Inc., Waltham, MA, USA) at a wavelength of $260 \mathrm{~nm}$. Total RNA (500 ng) was reverse transcribed in a final volume of $50 \mu \mathrm{l}$ using PrimeScript ${ }^{\circledR} \mathrm{RT}$ Reagent kit (Takara Bio, Inc., Otsu, Japan), according to the manufacturer's instructions. The cDNA was subsequently stored at $-20^{\circ} \mathrm{C}$.

Gene selection and primer design. A total of 12 genes belonging to various functional classes, which are frequently used as references genes in RT-qPCR studies, were selected for experimentation. The following genes were included in the present study: ACTB, YWHAZ, HPRT, TFRC, SDHA, B2M, $P G K 1, G A P D H, G 6 P D, R P L 19, R P S 24$ and $18 S$. The primer sequences are specified in Table I (Augct, Beijing, China). The primer sequences for $P G K 1, S D H A$, and $G 6 P D$ were based on previous publications $(7,9)$.

$R T-q P C R$. All RT-qPCR reactions were performed in a $20 \mu 1$ volume using SYBR ${ }^{\circledR}$ Premix Ex Taq ${ }^{\mathrm{TM}}$ II (Takara Bio, Inc.). The RT-qPCR was performed according to the instructions of the ABI 7500 real-time PCR system (Applied Biosystems Life Technologies, Foster City, CA, USA), which were as follows: Holding stage, $95^{\circ} \mathrm{C}$ for $3 \mathrm{~min}$; 40 cycles of $95^{\circ} \mathrm{C}$ for $15 \mathrm{sec}$ and $55^{\circ} \mathrm{C}$ for $34 \mathrm{sec}$; melt curve stage, $95^{\circ} \mathrm{C}$ for $15 \mathrm{secs}, 60^{\circ} \mathrm{C}$ for $1 \mathrm{~min}, 95^{\circ} \mathrm{C} 30 \mathrm{sec}$ and $60^{\circ} \mathrm{C}$ for $15 \mathrm{sec}$. A melt curve was included at the end of each $\mathrm{RT}-\mathrm{qPCR}$ reaction.

Data analysis. RefFinder (http://www.leonxie.com/referencegene.php?type $=$ reference) was used in order to validate 


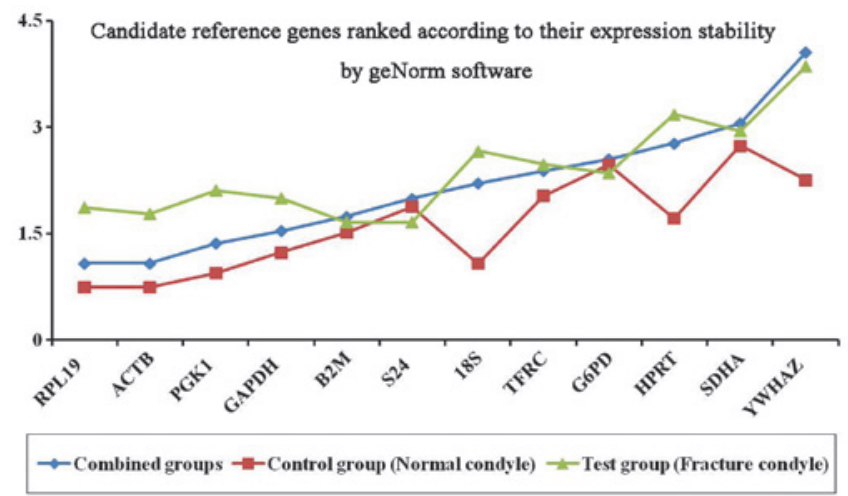

Figure 1. Candidate reference genes ranked according to their expression stability, as determined by geNorm. (Y-axis, stability values of the reference genes).

Table II. Expression stability of candidate reference genes, as determined by geNorm.

\begin{tabular}{|c|c|c|c|}
\hline \multirow[b]{2}{*}{ Gene name } & \multicolumn{3}{|c|}{ M-value } \\
\hline & Control & Test & Combined \\
\hline$A C T B$ & $0.745(1)$ & $1.778(3)$ & $1.081(1)$ \\
\hline RPL19 & $0.745(1)$ & $1.868(4)$ & $1.081(1)$ \\
\hline$P G K 1$ & $0.948(3)$ & $2.110(6)$ & $1.359(3)$ \\
\hline $18 S$ & $1.074(4)$ & $2.665(9)$ & $2.206(7)$ \\
\hline GAPDH & $1.235(5)$ & $2.001(5)$ & $1.535(4)$ \\
\hline$B 2 M$ & $1.518(6)$ & $1.657(1)$ & $1.743(5)$ \\
\hline$H P R T$ & $1.713(7)$ & $3.182(11)$ & $2.774(10)$ \\
\hline$S 24$ & $1.877(8)$ & $1.657(1)$ & $1.996(6)$ \\
\hline$T F R C$ & 2.037 (9) & $2.480(8)$ & $2.387(8)$ \\
\hline$Y W H A Z$ & $2.258(10)$ & $3.861(12)$ & $4.058(12)$ \\
\hline$G 6 P D$ & $2.473(11)$ & $2.350(7)$ & $2.553(9)$ \\
\hline$S D H A$ & $2.742(12)$ & $2.943(10)$ & 3.059 (11) \\
\hline
\end{tabular}

Control, normal ovine condyle; test, fractured ovine condyle; combined, both normal and fractured ovine condyle. Numbers in brackets represent ranking of genes by expression stability.

the reference genes. RefFinder is a user-friendly web-based comprehensive tool developed for evaluating and screening reference genes from extensive experimental datasets. RefFinder integrates the following currently available computational algorithms: geNorm, NormFinder, BestKeeper, and the comparative $\Delta \mathrm{Ct}$ method, in order to compare and rank experimental candidate reference genes. Based on the rankings from each program, RefFinder assigns an appropriate weight to an individual gene, and calculates the geometric mean of these weights in order to generate a final overall ranking. In addition to analyses of the test and control groups, the expression data of the two groups were also combined to form a combined group.

\section{Results}

Expression levels of reference genes. All 12 reference genes expressed in the mandibular condyle of the sheep exhibited a single marked increase in the melt-curve. RPL19 and 18S exhibited the highest expression levels, with a comparative threshold (Ct) mean of 17.46 and 26.09, respectively, whereas $B 2 M$ showed the lowest expression levels, with a $\mathrm{Ct}$ mean of 29.78. Ct refers to the cycle number at which the fluorescence intensity exceeds the set threshold. A high Ct value represents a low original template concentration, while a low $\mathrm{Ct}$ value represents a high original template concentration.

geNorm analysis. The stability values (M) of the reference genes were calculated using the geNorm algorithm. High M-values indicated high gene expression variability, whereas the most stable genes exhibited M-values <1.5. The M-values of the reference genes in the normal and fractured area of the sheep mandibular condyle as determined by geNorm, are shown in Table II and Fig. 1. In the control group, the most stable genes were RPL19 and ACTB, with an average M-value of 0.754 . The second most stable gene was $P G K 1$, with an average M-value of 0.948 , whereas the least stable genes were SDHA and G6PD. However, in the test group, the most stable genes were $S 24$ and $B 2 M$ with an average M-value of 1.657, whereas the least stable genes were HPRT and $Y W H A Z$. A comprehensive analysis of the combined group by geNorm identified the most stable genes to be RPL19 and ACTB, with an average M-value of 1.081. The second most stable gene in the combined group was $P G K 1$, with an average $\mathrm{M}$-value of 1.359 . The remaining genes exhibited high $\mathrm{M}$-values $>1.5$, with the least stable genes being SDHA and 
Table III. Expression stability of candidate reference genes, as determined by NormFinder.

\begin{tabular}{|c|c|c|c|}
\hline \multirow[b]{2}{*}{ Gene name } & \multicolumn{3}{|c|}{ Q-value } \\
\hline & Control & Test & Combined \\
\hline$A C T B$ & $0.838(1)$ & $1.642(4)$ & $1.252(2)$ \\
\hline$H P R T$ & $1.015(2)$ & 3.887 (11) & $2.933(10)$ \\
\hline $18 S$ & $1.073(3)$ & $2.851(9)$ & $2.266(7)$ \\
\hline RPL19 & $1.325(4)$ & $1.413(2)$ & $1.146(1)$ \\
\hline GAPDH & $1.439(5)$ & $2.110(7)$ & $1.884(6)$ \\
\hline$S 24$ & $1.675(6)$ & $2.086(6)$ & $2.468(8)$ \\
\hline$P G K 1$ & $1.696(7)$ & 1.209 (1) & $1.296(3)$ \\
\hline$T F R C$ & $1.937(8)$ & $1.928(5)$ & $1.723(5)$ \\
\hline$B 2 M$ & $2.064(9)$ & $2.233(8)$ & $1.679(4)$ \\
\hline$Y W H A Z$ & $2.832(10)$ & $6.895(12)$ & $8.789(12)$ \\
\hline$G 6 P D$ & $3.060(11)$ & $1.602(3)$ & $2.563(9)$ \\
\hline SDHA & $3.691(12)$ & $3.351(10)$ & 4.009 (11) \\
\hline
\end{tabular}

Control, normal ovine condyle; test, fractured ovine condyle; combined, both normal and fractured ovine condyle. Numbers in brackets represent ranking of genes by expression stability.

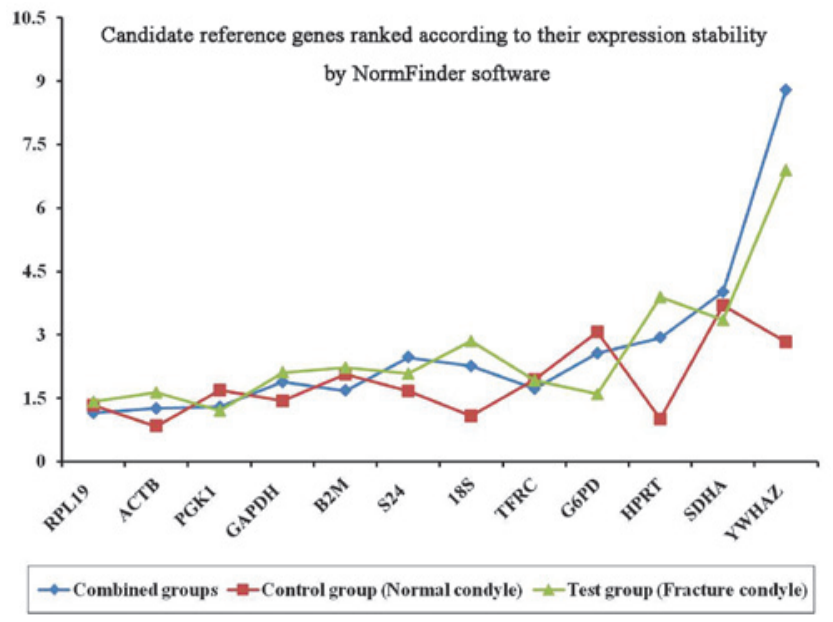

Figure 2. Candidate reference genes ranked according to their expression stability, as determined by NormFinder. (Y-axis, stability values of the reference genes).

$Y W H A Z$. Notably, SDHA and $Y W H A Z$ are considered conventional housekeeping genes in ovine blood (12).

NormFinder analysis. NormFinder ranks candidate genes according to their expression stability values (o) based on the similarity of their expression profiles. Lower values are assigned to the most stable genes. The candidate gene expression stabilities, as determined by NormFinder, are shown in Table III and Fig. 2. In the control group, the expression stability analysis demonstrated that $A C T B$ was the most stable gene, with a $\mathrm{Q}$-value of 0.838 . In the test group, $P G K 1$ was identified as the most stable reference gene, with a $\mathrm{Q}$-value of 1.209 . When the expression data were combined (combined group), the results confirmed that RPL19 was the most stable gene, with a Q-value of 1.146 .

BestKeeper analysis. Analysis of the reference gene expression levels was carried out using the BestKeeper software (Table IV and Fig. 3). In the control group, the results showed that $A C T B$ was the most stable gene, with a stability value of 0.78 . In the test group, $P G K 1$ was identified as the most stable reference gene, with a stability value of 0.93 . In the combined group, $P G K 1$ was determined to be the most stably expressed reference gene with a stability value of 0.96 , whereas $Y W A Z H$ was the least stable reference gene.

$\Delta C t$ analysis. The $\Delta C t$ method ranks a set of genes according to the repeatability of the gene expression differences among various samples (STDEV average). The results of the $\Delta \mathrm{Ct}$ analysis are shown in Table $\mathrm{V}$ and Fig. 4. In the control group, $A C T B$ was the most stable reference gene followed by $18 S$, with stability values of 2.07 and 2.21 , respectively. In the test group, G6PD and RPL19 were the highest stably expressed genes with stability values of 2.28 and 3.09 , respectively. The combined results (combined group) identified RPL19 and ACTB as the most stable genes, with stability values of 3.02 and 3.06, respectively. $Y W H A Z$ and $S D H A$ were the least stably expressed genes, with stability values of 9.05 and 4.88 , respectively. 
Table IV. Expression stability of candidate reference genes, as determined by BestKeeper.

\begin{tabular}{|c|c|c|c|}
\hline \multirow[b]{2}{*}{ Gene name } & \multicolumn{3}{|c|}{ Stability value } \\
\hline & Control & Test & Combined \\
\hline$A C T B$ & $0.78(1)$ & $1.39(2)$ & $1.18(2)$ \\
\hline $18 S$ & $0.87(2)$ & $2.51(9)$ & $1.88(6)$ \\
\hline$P G K 1$ & $1.00(3)$ & $0.93(1)$ & $0.96(1)$ \\
\hline RPL19 & $1.08(4)$ & $1.66(4)$ & $1.46(3)$ \\
\hline$G A P D H$ & $1.42(5)$ & $1.81(5)$ & $1.80(5)$ \\
\hline$S 24$ & $1.46(6)$ & $1.51(3)$ & $1.74(4)$ \\
\hline$H P R T$ & $1.50(7)$ & $4.16(12)$ & $2.69(10)$ \\
\hline$B 2 M$ & $1.98(8)$ & $2.28(8)$ & $2.16(9)$ \\
\hline TFRC & $2.07(9)$ & $2.16(7)$ & $1.94(7)$ \\
\hline$Y W H A Z$ & $2.23(10)$ & $3.23(11)$ & $4.93(12)$ \\
\hline G6PD & $3.56(11)$ & $1.92(6)$ & $2.14(8)$ \\
\hline$S D H A$ & $4.08(12)$ & $2.51(9)$ & $2.98(11)$ \\
\hline
\end{tabular}

Control, normal ovine condyle; test, fractured ovine condyle; combined, both normal and fractured ovine condyle. Numbers in brackets represent ranking of genes by expression stability.

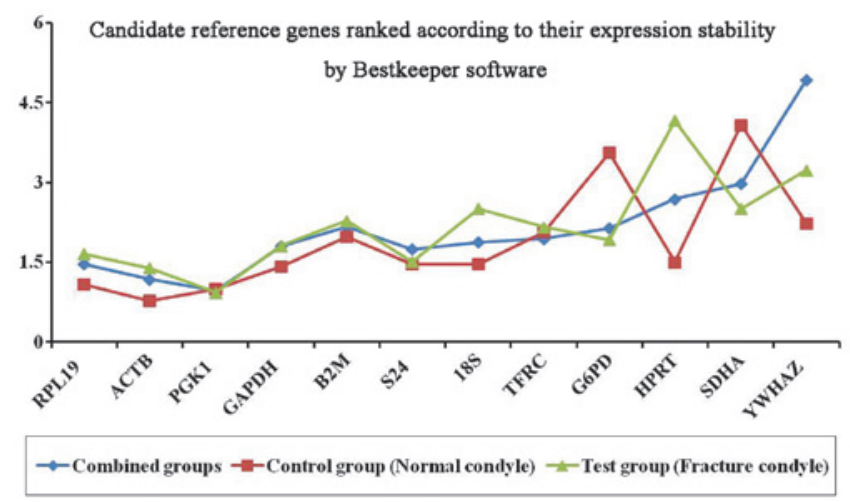

Figure 3. Candidate reference genes ranked according to their expression stability, as determined by BestKeeper. (Y-axis, stability values of the reference genes).

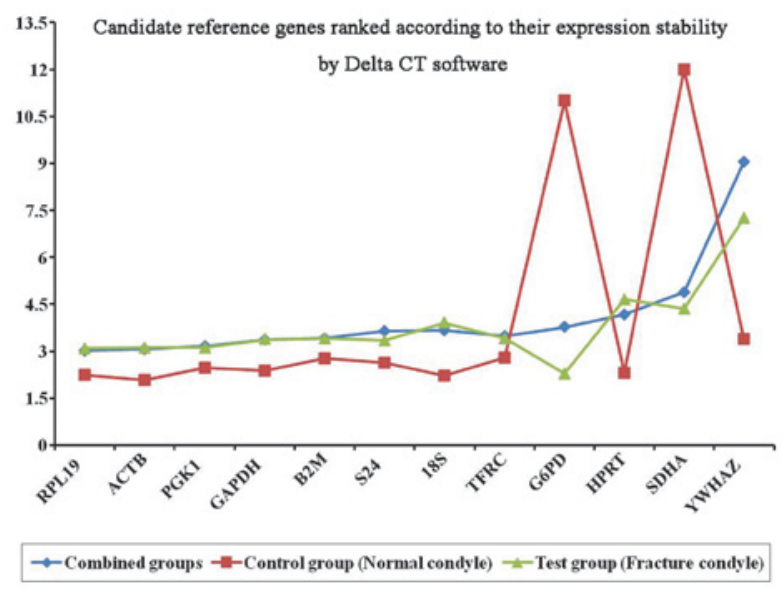

Figure 4. Candidate reference genes ranked according to their expression stability, as determined by the $\Delta \mathrm{Ct}$ method. (Y-axis, stability values of the reference genes).

Comprehensive analysis. The combined results obtained from geNorm, BestKeeper, the $\Delta \mathrm{Ct}$ method, and NormFinder were analyzed comprehensively using RefFinder (Table VI and Fig. 5). In the control group, RefFinder identified $A C T B$ as the most stably expressed gene, with a stability value of 1.00. In the test group, $P G K 1$ was the most stable reference gene with a stability value of 2.06 . The combined results of the two groups (combined group) identified RPL19 as the 
Table V. Expression stability of candidate reference genes, as determined by the $\Delta \mathrm{Ct}$ method.

\begin{tabular}{|c|c|c|c|}
\hline \multirow[b]{2}{*}{ Gene name } & \multicolumn{3}{|c|}{ Stability value } \\
\hline & Control & Test & Combined \\
\hline$A C T B$ & $2.07(1)$ & $3.11(2)$ & $3.06(2)$ \\
\hline $18 S$ & $2.21(2)$ & $3.91(9)$ & $3.67(8)$ \\
\hline RPL19 & $2.24(3)$ & 3.09 (1) & $3.02(1)$ \\
\hline$H P R T$ & $2.30(4)$ & $4.65(11)$ & $4.18(10)$ \\
\hline GAPDH & $2.38(5)$ & $3.38(6)$ & 3.37 (4) \\
\hline$P G K 1$ & $2.47(6)$ & $3.12(3)$ & $3.16(3)$ \\
\hline$S 24$ & $2.63(7)$ & $3.35(5)$ & $3.64(7)$ \\
\hline$B 2 M$ & $2.77(8)$ & $3.42(8)$ & $3.42(5)$ \\
\hline TFRC & $2.79(9)$ & $3.41(7)$ & $3.49(6)$ \\
\hline$Y W H A Z$ & $3.40(10)$ & $7.25(12)$ & $9.05(12)$ \\
\hline$G 6 P D$ & $11.00(11)$ & $2.28(4)$ & $3.77(9)$ \\
\hline$S D H A$ & $12.00(12)$ & $4.36(10)$ & $4.88(11)$ \\
\hline
\end{tabular}

Control, normal ovine condyle; test, fractured ovine condyle; combined, normal and fractured ovine condyle. Numbers in brackets represent ranking of genes by expression stability.

Table VI. Expression stability of candidate reference genes, as determined by RefFinder.

\begin{tabular}{|c|c|c|c|}
\hline \multirow[b]{2}{*}{ Gene name } & \multicolumn{3}{|c|}{ Stability value } \\
\hline & Control & Test & Combined \\
\hline$A C T B$ & $1.00(1)$ & $2.63(3)$ & $1.68(2)$ \\
\hline RPL19 & $2.63(2)$ & $2.38(2)$ & $1.32(1)$ \\
\hline $18 S$ & $2.63(3)$ & $9.00(9)$ & $6.96(8)$ \\
\hline$P G K 1$ & $4.41(4)$ & $2.06(1)$ & $2.28(3)$ \\
\hline$H P R T$ & $4.45(5)$ & $11.24(11)$ & $10.00(10)$ \\
\hline GAPDH & $5.00(6)$ & $5.69(7)$ & $4.68(4)$ \\
\hline$S 24$ & $6.70(7)$ & 3.08 (4) & $6.05(6)$ \\
\hline$B 2 M$ & $7.67(8)$ & $4.76(6)$ & $5.48(5)$ \\
\hline$T F R C$ & $8.74(9)$ & $6.65(8)$ & $6.40(7)$ \\
\hline$Y W H A Z$ & $10.00(10)$ & $11.74(12)$ & $2.00(12)$ \\
\hline$G 6 P D$ & $11.00(11)$ & $4.74(5)$ & $8.74(9)$ \\
\hline SDHA & $12.00(12)$ & $10.00(10)$ & $11.00(11)$ \\
\hline
\end{tabular}

Control, normal ovine condyle; test, fractured ovine condyle; combined, both normal and fractured ovine condyle. Numbers in brackets represent ranking of genes by expression stability.

most stably expressed reference gene with a stability value of 1.32 , whereas $Y W A Z H$ was the least stable reference gene. Candidate reference genes of the control group, test group and combined group, ranked using five different methods, were compared in Figs. 6-8, respectively. Though each algorithm employs a different method to determine the most suitable reference gene, the results obtained from all of the methods exhibited a similar trend.

\section{Discussion}

Due to its high specificity, sensitivity, efficiency and repetitiveness, RT-qPCR has been widely used to measure the expression levels of target genes. The advantages of RT-qPCR also includes rapid detection, simple design, and low cost. However, the primary limitation of relative quantification is that a reference gene must be included as an internal control. Numerous reference genes, such as $G A P D H, A C T B, 18 S$, and $G 6 P D$, have previously been identified. However, to date, no reference gene has been shown to be constitutively expressed in all cell types and under all experimental conditions. Therefore, evaluating the expression stability of candidate reference genes and identifying the most stable gene is important in order to ensure the authenticity and reliability of a given study. Numerous reference genes have been identified as suitable for various ovine tissue samples. SDHA and $Y W H A Z$ are considered to be the most suitable internal controls for RT-qPCR analysis of ovine blood (12), ACTB for the cerebrum and spleen, and 


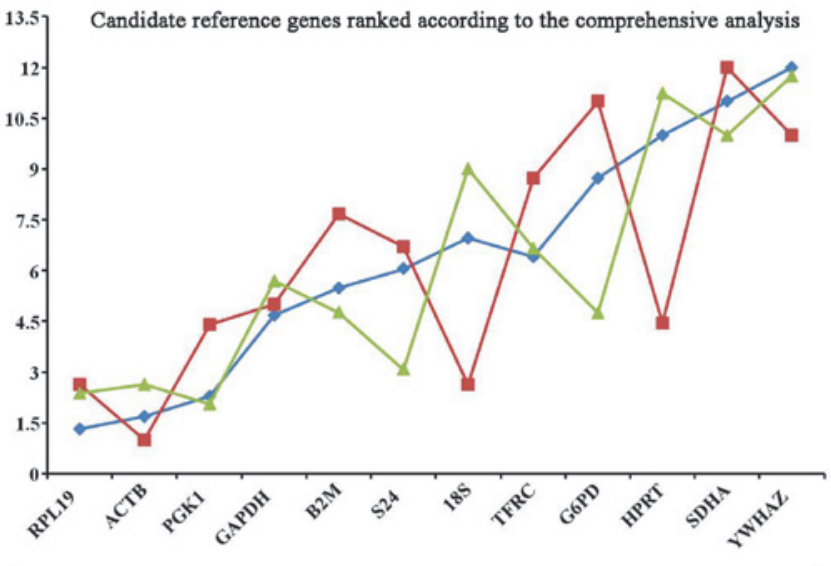

$\rightarrow$ Combined groups $\rightarrow-$-Control group (Normal condyle) $\rightarrow-$ Test group (Fracture condyle)

Figure 5. Candidate reference genes ranked, as determined by comprehensive analysis. (Y-axis, stability values of the reference genes)

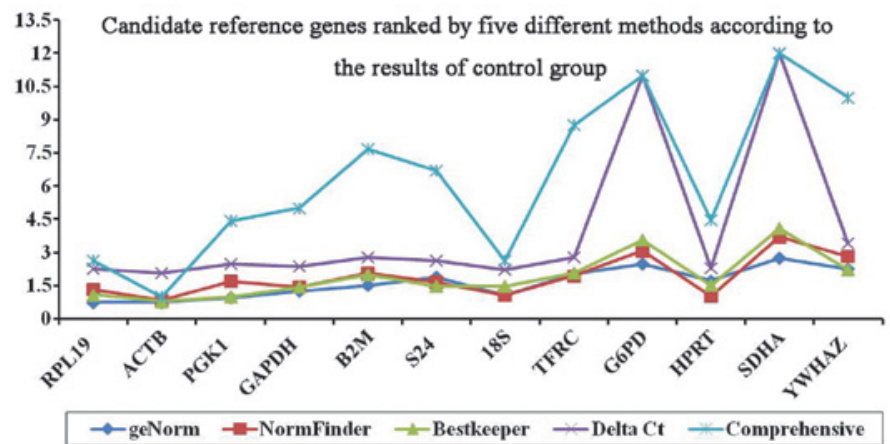

Figure 6. Candidate reference genes of the control group ranked using five different methods. (Y-axis, stability values of the reference genes).

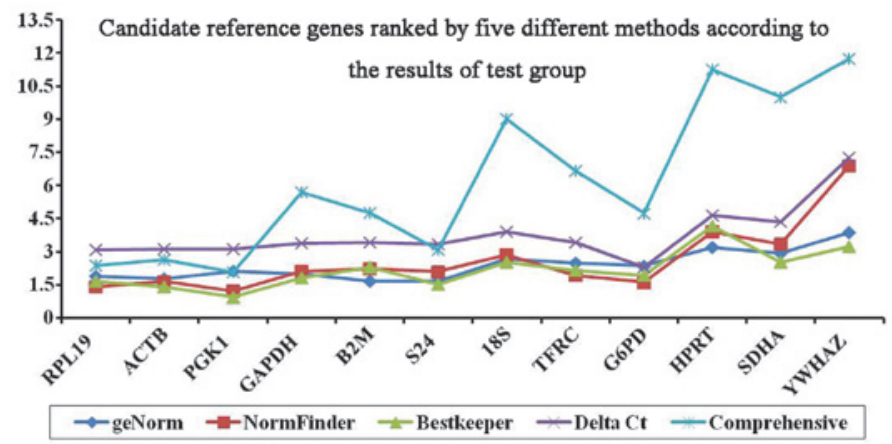

Figure 7. Candidate reference genes of the test group ranked using five different methods. (Y-axis, stability values of the reference genes).

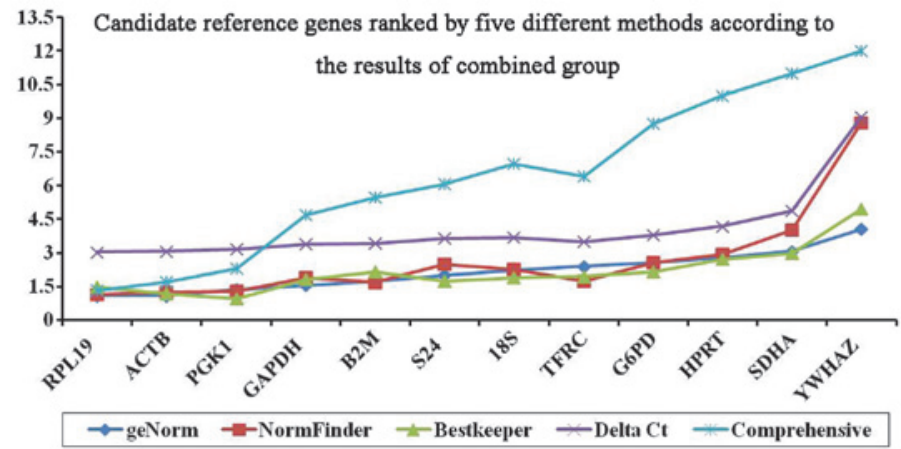

Figure 8. Candidate reference genes of the combined group ranked using five different methods. (Y-axis, stability values of the reference genes). 
GAPDH for the mesenteric lymph node (7). However, little is currently known regarding the most suitable reference gene for the bone, particularly for the sheep mandibular condyle. Sheep have an important role in the study of human bone diseases, particularly studies associated with temporomandibular diseases $(13,14)$. Therefore, the identification of a reference gene suitable for the normalization of sheep mandibular condyle RT-qPCR analyses is necessary.

In the present study, the expression stability of 12 housekeeping genes were examined in both normal and fractured sheep mandibular condyles using the following algorithms: geNorm, NormFinder, BestKeeper, and the $\Delta \mathrm{Ct}$ method. geNorm (15) calculates the expression ratio of two ideal internal control genes in two identical samples, regardless of the experimental conditions or cell type. For each control gene, the pairwise variation is determined as the standard deviation of the logarithmically transformed expression ratios, and the internal control gene-stability value (M) is determined as the average pairwise variation of a particular gene with all other control genes. NormFinder (3) ranks the candidate genes according to their expression stability values (o) based on the similarity of their expression profiles. BestKeeper (16) identifies the most suitable reference genes using a pairwise correlation analysis of all pairs of candidate genes, and calculates the geometric mean of the most suitable genes. The $\Delta \mathrm{Ct}$ (17) method ranks a set of genes according to the repeatability of the gene expression differences among various samples (average of STDEV). Although each algorithm employs a different method to determine the most suitable reference gene, in the present study the results obtained from all five methods exhibited a similar trend (Fig. 6-8). All methods determined that RPL19, ACTB, and PGK1 were the most stably expressed reference genes in the sheep mandibular condyle, regardless of whether the candidate genes were analyzed for the normal group, the fracture group, or the combined group. The results obtained from the various algorithms were comprehensively analyzed using RefFinder. Based on the rankings obtained from each algorithm, RefFinder assigns an appropriate weight to an individual gene, and calculates the geometric mean of these weights, in order to obtain an overall final ranking. The results demonstrated that $A C T B$ and RPL19 were the most stable reference genes expressed in the normal sheep mandibular condyle, PGK1 and RPL19 were the most stable reference genes expressed in the fractured sheep mandibular condyle, whereas RPL19 and ACTB were the most stably expressed reference genes in both the normal and fractured sheep mandibular condyle (combined group). The results of the present study identified RPL19 as the most suitable reference gene for studies associated with the sheep mandibular condyle. In addition, $A C T B$ and $P G K 1$ were shown to be suitable alternatives. These results differ from previous reports of sheep reference gene suitability, which included the identification of SDHA and $Y W H A Z$ as the most stable reference genes for ovine blood (12), ACTB for the cerebrum and spleen, and $G A P D H$ for the mesenteric lymph node (7). In conclusion, the results of the present study suggested that if these previously reported genes were to be used as reference genes in studies associated with the sheep mandibular condyle, the results of such a study may be negatively affected.

\section{Acknowledgements}

The present study was supported by a grant from the National Natural Science Foundation of China (grant no. 81271168).

\section{References}

1. Heid CA, Stevens J, Livak KJ and Williams PM: Real time quantitative PCR. Genome Res 6: 986-994, 1996.

2. Bustin SA: Quantification of mRNA using real-time reverse transcription PCR (RT-PCR): Trends and problems. J Mol Endocrinol 29: 23-39, 2002.

3. Andersen CL, Jensen JL and Ørntoft TF: Normalization of real-time quantitative reverse transcription-PCR data: A model-based variance estimation approach to identify genes suited for normalization, applied to bladder and colon cancer data sets. Cancer Res 64: 5245-5250, 2004.

4. Chari R, Lonergan KM, Pikor LA, Coe BP, Zhu CQ, Chan TH, MacAulay CE, Tsai MS, Lam S, Ng RT and Lam WL: A sequence-based approach to identify reference genes for gene expression analysis. BMC Med Genomics 3: 32, 2010.

5. Selvey S, Thompson EW, Matthaei K, Lea RA, Irving MG and Griffiths LR: Beta-actin - an unsuitable internal control for RT-PCR. Mol Cell Probes 15: 307-311, 2001.

6. Ohl F, Jung M, Xu C, Stephan C, Rabien A, Burkardt M, Nitsche A, Kristiansen G, Loening SA, Radonić A and Jung K: Gene expression studies in prostate cancer tissue: Which reference gene should be selected for normalization? J Mol Med (Berl) 83: 1014-1024, 2005.

7. Garcia-Crespo D, Juste RA and Hurtado A: Selection of ovine housekeeping genes for normalisation by real-time RT-PCR; analysis of PrP gene expression and genetic susceptibility to scrapie. BMC Vet Res 1: 3, 2005.

8. Lampo E, Van Poucke M, Vandesompele J, Erkens T, Van Zeveren A, and Peelman LJ: Positive correlation between relative mRNA expression of PRNP and SPRN in cerebral and cerebellar cortex of sheep. Mol Cell Probes 23: 60-64, 2009.

9. Passmore M, Nataatmadja M and Fraser JF: Selection of reference genes for normalisation of real-time RT-PCR in brain-stem death injury in Ovis aries. BMC Mol Biol 10: 72, 2009.

10. Lyahyai J, Serrano C, Ranera B, Badiola JJ, Zaragoza P, and Martin-Burriel I: Effect of scrapie on the stability of housekeeping genes. Anim Biotechnol 21: 1-13, 2010.

11. Liu CK, Liu P, Meng FW, Deng BL, Xue Y, Mao TQ and Hu KJ: The role of the lateral pterygoid muscle in the sagittal fracture of mandibular condyle (SFMC) healing process. Br J Oral Maxillofac Surg 50: 356-360, 2012.

12. Peletto S, Bertuzzi S, Campanella C, Modesto P, Maniaci MG, Bellino C, Ariello D, Quasso A, Caramelli M and Acutis PL: Evaluation of internal reference genes for quantitative expression analysis by real-time PCR in ovine whole blood. Int J Mol Sci 12: 7732-7747, 2011.

13. Meng FW, Hu KJ, Kong L, Zhao YT, Liu YP and Zhou SX: Morphological evaluation of temporomandibular joint after open and closed treatment of type B diacapsular condylar fractures in sheep. Ann Anat 191: 288-293, 2009.

14. Takaishi M, Kurita K, Hatano Y, Matsuura H, Borg M and Goss NA: Effects of postoperative radiotherapy for temporomandibular joint ankylosis after gap arthroplasty: An animal study using sheep. J Oral Maxillofac Surg 68: 1763-1769, 2010.

15. Vandesompele J, De Preter K, Pattyn F, Poppe B, Van Roy N, De Paepe A and Speleman F: Accurate normalization of real-time quantitative RT-PCR data by geometric averaging of multiple internal control genes. Genome Biol 3: RESEARCH0034, 2002.

16. Pfaffl MW, Tichopad A, Prgomet C and Neuvians TP: Determination of stable housekeeping genes, differentially regulated target genes and sample integrity: BestKeeper - Excel-based tool using pair-wise correlations. Biotechnol Lett 26: 509-515, 2004.

17. Silver N, Best S, Jiang J and Thein SL: Selection of housekeeping genes for gene expression studies in human reticulocytes using real-time PCR. BMC Mol Biol 7: 33, 2006. 